\title{
Transatlantica
}

Revue d'études américaines. American Studies Journal

Hors-série | 2021

Numéro anniversaire : 20 ans de la revue

\section{Entretien avec Ana Lucia Araujo}

\section{Michaël Roy et Ana Lucia Araujo}

\section{(2) OpenEdition}

Journals

Édition électronique

URL : https://journals.openedition.org/transatlantica/17480

DOI : $10.4000 /$ transatlantica. 17480

ISSN : 1765-2766

Éditeur

Association française d'Etudes Américaines (AFEA)

Référence électronique

Michaël Roy et Ana Lucia Araujo, «Entretien avec Ana Lucia Araujo », Transatlantica [En ligne], Horssérie | 2021, mis en ligne le 01 octobre 2021, consulté le 11 octobre 2021. URL : http://

journals.openedition.org/transatlantica/17480; DOI : https://doi.org/10.4000/transatlantica.17480

Ce document a été généré automatiquement le 11 octobre 2021.

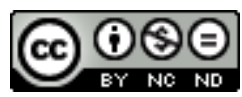

Transatlantica - Revue d'études américaines est mise à disposition selon les termes de la licence Creative Commons Attribution - Pas d'Utilisation Commerciale - Pas de Modification 4.0 International. 


\title{
Entretien avec Ana Lucia Araujo
}

\author{
Michaël Roy et Ana Lucia Araujo
}

Michaël Roy (M.R.) : Bonjour Ana. Vous avez rejoint le comité scientifique de Transatlantica tout récemment: bienvenue! Pouvez-vous vous présenter pour les lectrices et lecteurs de la revue?

Ana Lucia Araujo (A.L.A.) : Je suis une historienne dont les travaux de recherche se concentrent sur l'histoire et la mémoire de l'esclavage et de la traite atlantique des esclaves. J'ai une double formation en histoire et histoire de l'art. Mes recherches ont d'abord porté sur la culture visuelle de l'esclavage et la mémoire de l'esclavage issue des échanges entre le Brésil et le golfe du Bénin. Depuis que je suis arrivée aux ÉtatsUnis, je m'intéresse aussi à ce pays dans le cadre de mes travaux sur la mémoire de l'esclavage. De manière générale, parce que l'esclavage et la traite atlantique ont opéré par-delà les frontières nationales, je travaille à partir d'une perspective comparative et transnationale. Depuis 2008, j'enseigne à Howard University, à Washington, DC, où je suis actuellement professeure titulaire. Je suis aussi membre du comité scientifique du projet « La Route de l'esclave : résistance, liberté, héritage » de l'UNESCO.

M.R. : Vous êtes en poste dans une université " historiquement noire » (historically Black colleges and universities). Est-ce un choix de votre part? Pouvez-vous nous parler de votre expérience dans cet établissement?

A.L.A. : J'ai candidaté à un poste à Howard University parce que j'avais des affinités avec cet établissement du fait de mon travail de recherche. Howard s'est donné pour mission d'enseigner l'histoire des populations de la diaspora africaine, donc en ce sens c'est d'abord l'institution qui m'a choisie pour occuper ce poste et qui m'a ensuite permis d'accéder au rang de professeure associée (associate professor) puis de professeure titulaire (full professor). Mais c'est aussi moi qui ai choisi Howard University. Ici, aux États-Unis, les enseignants-chercheurs et enseignanteschercheuses changent fréquemment d'établissement. J'ai opté pour cette université pour un ensemble de raisons, mais principalement parce qu'à Howard University j'ai toute liberté pour mener mes travaux de recherche et la possibilité de faire cours à des étudiantes et étudiants passionnés. 
M.R. : Pouvez-vous nous parler également de votre expérience de chercheuse d'origine étrangère aux États-Unis ? Est-ce que cette position vous conduit à adopter un regard ou une approche différentes de chercheuses et chercheurs états-uniens travaillant sur les enjeux de mémoire?

A.L.A. : Je suis d'origine étrangère depuis longtemps, d'abord au Canada où j'ai fait l'essentiel de mes études. Sans doute mon approche est-elle différente dans la mesure où mon travail ne porte pas uniquement sur les États-Unis ou sur un pays européen, comme c'est le cas d'une majorité d'historiens nord-américains. Par ailleurs, beaucoup de chercheurs aux États-Unis sont monolingues, ce qui les empêche de lire en langue étrangère ou de faire de la recherche sur d'autres espaces géographiques, et donc de sortir d'un récit exceptionnaliste de l'histoire états-unienne. En venant d'ailleurs, on est plus facilement amenée à adopter des perspectives auxquelles des chercheurs « locaux » n'auraient pas pensé, à relier entre elles des réalités qui, au premier regard, semblent incompatibles; cela permet de gagner en nuance. D'ailleurs, ma position me permet aussi de voir l'histoire du Brésil d'un œil différent de celui que j'aurais si j'étais restée dans ce pays.

M.R. : Votre approche se caractérise par sa dimension transnationale: dans votre dernier livre, Slavery in the Age of Memory, vous vous intéressez aux mémoires de l'esclavage aux États-Unis, mais aussi au Bénin, au Brésil, en Angleterre et en France. Que permet une telle approche ? Pose-t-elle certaines difficultés théoriques ou pratiques?

A.L.A.: Mon objet d'étude impose une telle approche. L'esclavage et la traite ont émergé à partir de la " première " globalisation issue des grandes navigations et des rencontres entre Européens et Africains au $\mathrm{Xv}^{\mathrm{e}}$ siècle. Je crois qu'une approche nationale pose de très grands obstacles pour l'étude de phénomènes qui ont existé du fait d'échanges ayant eu lieu dans une sphère internationale. De plus, mes recherches montrent que la réémergence de la mémoire de l'esclavage au cours des trois dernières décennies a eu lieu à peu près en même temps dans ces différents pays. Bien sûr, il importe de mettre aussi en valeur les spécificités nationales. Combiner macrohistoire et microhistoire pose souvent des difficultés, avec une question récurrente : faut-il privilégier les éléments en commun ou les traits distinctifs?

M.R. : Dans Slavery in the Age of Memory, vous portez un regard plutôt critique sur diverses entreprises de mémorialisation de l'esclavage dans l'espace public, les musées, etc. Pouvez-vous nous donner un exemple de mémorial, de musée ou de statue dédiée à la mémoire de l'esclavage, aux États-Unis par exemple, et qui vous semble problématique?

A.L.A. : De façon générale, tous ces dispositifs sont problématiques. Ceux qui sont les plus problématiques n'ont pas été originellement dédiés à l'esclavage et aux personnes asservies, mais à des abolitionnistes par exemple. Je pense notamment à l'Emancipation Memorial, un monument érigé en 1876 à Lincoln Park, dans la capitale états-unienne. Ce monument représente Lincoln en " grand émancipateur ", debout, une main posée sur la Proclamation d'émancipation de 1863, l'autre main tendue, comme s'il donnait la liberté à l'esclave qui est agenouillé devant lui. C'est une représentation très paternaliste et très réductrice du processus d'émancipation.

M.R. : À l'inverse, y a-t-un mémorial, un musée, une statue qui vous paraît particulièrement « réussie»?

A.L.A.: La Whitney Plantation, en Louisiane, me semble particulièrement intéressante. 
M.R. : En quoi ? Se différencie-t-elle d'autres musées ou plantations comme Monticello ou Mount Vernon?

A.L.A. : Les plantations de Monticello et Mount Vernon ont respectivement appartenu aux présidents Jefferson et Washington et ont été patrimonialisées pour mettre en valeur la mémoire de ces deux présidents qui étaient aussi esclavagistes. La Whitney Plantation a été achetée par l'homme d'affaires John Cummings, dont l'objectif était de mettre en valeur l'histoire des personnes réduites en esclavage dans la plantation plutôt que de raconter et de glorifier l'histoire de ses anciens propriétaires. C'est d'ailleurs l'une des seules plantations du Sud des États-Unis où la maison des propriétaires ne se visite pas.

M.R. : Slavery in the Age of Memory a été écrit juste avant la séquence du printemps 2020, qui, à la suite de la mort de George Floyd, a vu d'importantes mobilisations sociales et le déboulonnage d'un certain nombre de statues à l'effigie de personnalités ayant défendu l'esclavage et la colonisation, dans le monde anglophone en tout cas. Quel regard portezvous sur cette séquence et sur les déboulonnages qui ont eu lieu depuis?

A.L.A. : Le livre montre que ce mouvement était déjà en marche depuis 2015 avec Rhodes Must Fall, une campagne qui a commencé en Afrique du Sud puis s'est développée en Grande-Bretagne. Bien entendu, ce mouvement s'inscrivait aussi dans le prolongement de Black Lives Matter, dont les militants avaient, dès 2013, lancé des débats et mené des actions autour des monuments confédérés et d'autres marqueurs associés au passé esclavagiste. La séquence du printemps 2020 n'est pas tant une éruption soudaine et imprévisible qu'un moment culminant où ces revendications communes transnationales, déjà exprimées par le passé, ont gagné en visibilité. La crise du COVID-19 a sans aucun doute joué un rôle de catalyseur dans ce processus, tout d'abord aux États-Unis où un grand nombre de personnes ont participé aux manifestations, puis en Angleterre, et à plus petite échelle au Portugal, en Belgique et en France. La pandémie a affecté de manière disproportionnée les populations racisées et, par ailleurs, beaucoup de citoyens étaient chez eux à l'époque, et peutêtre plus attentifs aux revendications; tout cela a pu contribuer à l'ampleur du mouvement.

M.R. : Le mouvement ne semble pas vraiment avoir pris en France. Comment expliquezvous ces résistances?

A.L.A. : Je crois que malgré certaines difficultés, le débat sur la mise en mémoire et la patrimonialisation de l'esclavage a commencé en France il y a au moins vingt ans, et qu'il s'est poursuivi au printemps 2020. La situation dans les anciens ports esclavagistes français me paraît un peu plus avancée qu'en Grande-Bretagne, notamment à Bristol, ou la statue d'Edward Colston a été déboulonnée au printemps 2020. Paris, à la différence de Londres, n'était pas un port esclavagiste, il y a donc moins de symboles associés à l'esclavagisme et au colonialisme. Malgré l'absence d'un mémorial national et d'un musée national, la France a lancé un certain nombre d'initiatives pour commémorer la mémoire de l'esclavage, issues de la loi Taubira, dont la journée nationale du 10 mai et la création de la Fondation pour la mémoire de l'esclavage. Emmanuel Macron (quoi qu'on pense de son programme politique) a commandé à Felwine Sarr et Bénédicte Savoy un «Rapport sur la restitution du patrimoine culturel africain "; la restitution d'un petit nombre de pièces pillées lors de la conquête coloniale de l'Afrique est maintenant en marche. Il ne faut pas oublier non plus qu'à Fort-de-France une statue de Joséphine de Beauharnais a été déboulonnée en juillet 2020, et qu'avant cela, en mai 2020, deux statues de Victor 
Schœlcher avaient été déjà abattues en Martinique. Les activistes qui ont mené ces actions ont écrit un manifeste dans lequel ils évoquaient des notions telles que celles d'appropriation et de réparation. Des statues de Schœlcher ont également été renversées en Guadeloupe et en Guyane. Il n'est donc pas tout à fait exact de dire que le mouvement n'a pris en France : la Martinique, la Guadeloupe et la Guyane font bien partie de la France.

M.R.: Vous avez aussi beaucoup travaillé sur les réparations. Que pensez-vous des initiatives mises en place dans certaines universités états-uniennes et britanniques pour mettre en lumière leur passé esclavagiste? C'est notamment le cas à Georgetown University, près de chez vous. Est-ce que ce travail sur le passé peut être en soi compris comme une forme de réparation symbolique? Voyez-vous certaines limites à ce genre d'initiatives?

A.L.A. : C'est un travail de réparation symbolique, dans le cas de Georgetown et dans le cas d'autres universités comme Brown (dans le Rhode Island) et l'université de Virginie. Seule l'université de Brown a mis en place un programme de réparations financières, qui prend la forme de bourses ou d'aides spécifiques accordées aux étudiantes et étudiants issus des minorités noires. Dans la plupart des cas, les établissements se contentent de mettre en place des groupes de travail ayant pour tâche d'examiner les liens historiques de l'université avec l'esclavage, mais ne font rien pour lutter contre les inégalités présentes liées au racisme et à la discrimination des étudiantes et étudiants racisés. L'effet de ces initiatives est donc limité. Il y a une dimension de communication. Et il s'agit davantage de répondre aux revendications étudiantes que de s'attaquer à la nature élitiste et raciste des institutions en question.

M.R. : J'aimerais vous interroger sur votre usage du numérique et des réseaux sociaux. Pouvez-vous nous présenter le \#slaveryarchive Book Club que vous organisez en ligne ? Sa création est-elle liée au contexte pandémique? Comment faites-vous votre programmation? Êtes-vous satisfaite du résultat?

A.L.A. : J'ai créé le Book Club pour briser l'isolement créé par la pandémie dans un moment où de nombreux historiens et universitaires, dont je suis, publient des livres mais se retrouvent sans la possibilité d'en faire la promotion en personne. J'ai d'abord eu l'idée, puis je l'ai partagée avec l'historienne Jessica Marie Johnson, qui, comme moi, utilise beaucoup les réseaux sociaux comme instrument de travail et outil des humanités numériques. Après une première séance, il était clair que nous aurions besoin de plus de personnes dans l'équipe ; nous avons alors invité Alex Gil, qui est spécialisé en humanités numériques, et Vanessa Holden, historienne de l'esclavage avec laquelle nous collaborons depuis longtemps.

La programmation se fait à partir d'un critère principal : les livres présentés portent sur l'histoire de l'esclavage, de la traite des esclaves et de la diaspora africaine, notamment dans le monde atlantique. Au départ nous contactions les collègues que nous connaissions, mais le Book Club commence à avoir une certaine présence sur les réseaux sociaux et ce sont parfois des collègues qui nous contactent; nous avons inclus leurs travaux lorsqu'ils nous intéressaient. Nous organisons autant de séances que possible, mais pendant cette deuxième année, nous avons privilégié les livres écrits par des femmes, qui souvent hésitent à nous contacter spontanément.

Nous sommes très heureuses du résultat. C'est beaucoup de travail, mais cela a permis de créer une communauté active de gens issus de pays différents, qui ne se seraient peut-être pas rencontrés autrement. 
M.R. : Pouvez-vous nous expliquer le principe du mot-dièse \#slaveryarchive que vous avez créé sur Twitter? Est-ce que vous considérez ce volet de votre activité comme une forme d'histoire publique (public history)?

A.L.A. : Il y a plus de cinq ans, alors que les débats sur le passé esclavagiste du pays refaisaient surface dans la sphère publique aux États-Unis et que je préparais mon livre Slavery in the Age of Memory, j'ai créé le mot-dièse \#slaveryarchive pour indexer des contenus liés à l'histoire et à la mémoire de l'esclavage atlantique: livres, colloques, création de monuments, découverte de cimetières, etc. Je faisais des recherches quotidiennes sur internet et publiais des tweets renvoyant vers les pages en question, tout en utilisant le mot-dièse \#slaveryarchive; grâce à l'application IFTTT, les tweets étaient automatiquement référencés sur un site Tumblr. Au fil des années, j'ai formé une base de données numérique à partir de ces contenus, qui d'ailleurs ne sont pas qu'en anglais, mais aussi en français, portugais et espagnol. Même si je ne suis plus très systématique dans la collecte de données, le Tumblr \#slaveryarchive donne une bonne idée de tout ce qui se passe autour de la mémoire de l'esclavage.

Je conçois effectivement cela comme une forme d'histoire publique, car le mot-dièse peut être suivi par n'importe qui. Cela permet de rendre l'histoire de l'esclavage accessible à un large public.

M.R. : Vous utilisez également Twitter pour interpeller certains organisateurs de colloques, séminaires, etc., sur le manque de représentation des femmes dans leurs événements. Pouvez-vous nous en parler?

A.L.A. : Je me sers des réseaux sociaux pour promouvoir les travaux de recherche produits par des femmes. Quand je vois passer un colloque, un panel, un ouvrage collectif où les femmes sont absentes ou sous-représentées, je le signale. J’espère ainsi encourager mes collègues à promouvoir elles-mêmes leurs travaux.

M.R. : Puisque nous fêtons les vingt ans de Transatlantica, je voulais terminer en vous interrogeant sur votre rapport aux revues scientifiques. Êtes-vous impliquée dans d'autres revues, d'autres comités scientifiques? Quel regard portez-vous sur la publication de revues scientifiques aujourd'hui, sachant que beaucoup de ces revues sont protégées par des pare-feu et inaccessibles pour le grand public ?

A.L.A.: Oui, je fais partie des comités scientifiques de plusieurs revues universitaires aux États-Unis, en France, en Angleterre, en Colombie et au Portugal. Je suis notamment membre pour trois ans du comité de rédaction de l'American Historical Review, ce qui prend beaucoup de temps : je dois lire et évaluer environ deux articles par mois. Nous avons bien sûr besoin de ces revues, mais elles posent certains problèmes récurrents, à commencer par le temps souvent long entre la soumission d'article et la publication, le petit nombre de lecteurs, les difficultés d'accès. Beaucoup de bibliothèques universitaires ne s'abonnent plus aux revues, qui sont de plus en plus chères, au point que même certaines chercheuses et chercheurs rencontrent des problèmes d'accès.

M.R. : Avez-vous une revue, un livre récent que vous voudriez nous conseiller?

A.L.A. : C'est difficile, car il y a beaucoup de très bon ouvrages parus récemment, mais je recommande le nouveau livre de l'historienne Tiya Miles, All That She Carried: The Journey of Ashley's Sack, a Black Family Keepsake (Random House, 2021). C'est un bijou : l'autrice part d'un objet singulier pour raconter une histoire émouvante où il est question d'esclavage, d'émancipation et de post-esclavage aux États-Unis. 
M.R. : C'est bien noté. Merci Ana!

\section{AUTEURS}

MICHAËL ROY

Université Paris Nanterre

ANA LUCIA ARAUJO

Howard University 